\section{NREL Develops Sub-Hour Solar Power Data Set}

\author{
NREL data will help utilities incorporate solar energy into \\ their electric power systems.
}

Large-scale deployment of solar energy requires a favorable environment for interconnecting and operating solar power plants. But realizing high volumes and economies of scale will be difficult if solar penetrations are limited by reliability, interconnection, and operational rules and requirements. Utilities need tools and data to study and enable high solar penetrations on their power systems.

Sub-hourly solar power output data sets with realistic spatial and temporal correlations are necessary to answer questions about how high penetrations of solar impact the grid and what options, such as increased reserve requirements, may be needed to mitigate the variability and uncertainty of solar power. The National Renewable Energy Laboratory (NREL) has developed a methodology to synthesize 1-minute power output from photovoltaic and concentrating solar power plants of various sizes. Researchers measure global horizontal irradiance (GHI) at 10 sites (including Boulder and Golden, Colorado) and hourly snapshot satellite image-derived irradiance values. For each hour, the observed GHI value for a satellite grid cell of interest and surrounding grid cells is related, via probability distributions, to one of five temporal cloud-coverage classifications (Class I, II, III, IV, or V). Each class of cloudcoverage variability has a separate algorithm to select 1-minute time-step GHI values. Sub-hour GHI values are integrated with other meteorological data to generate an input file for solar power conversion via the System Advisor Model.

Using spatial variability as an input to the sub-hour solar power algorithm generates a data set with appropriate spatial-temporal ramping correlation statistics. The resulting individual site simulated time series shows a remarkable degree of similarity to the measured time series for the same site. A large-scale data set (1,117 locations) is being used in the Western Wind and Solar Integration Study Phase 2, Demand Response and Storage Integration Study, and studies undertaken at the Western Electricity Coordinating Council.

Technical Contact: Marissa Hummon, marissa.hummon@nrel.gov

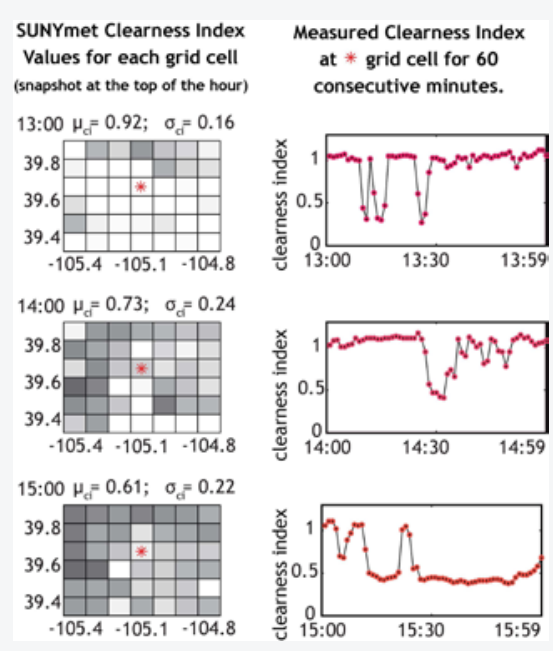

Variability in the clearness index-a ratio of the actual-to-expected insolation -is visualized as a snapshot in time across a region (left). NREL relates the spatial variability to time series irradiance measurements (right) at a single site (red star).

\section{Key Research Results}

\section{Achievement}

NREL developed a methodology to synthesize 1-minute power outputs from photovoltaic and concentrating solar power plants of various sizes.

\section{Key Result}

Individual site simulated time series shows a remarkable degree of similarity to measured time series for the same site.

\section{Potential Impact}

Utilities will be able to better understand how large solar power plants will affect their systems, how to plan for that, and what steps to take to mitigate the integration of solar power into the grid.

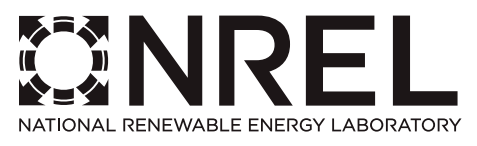

15013 Denver West Parkway | Golden, CO 80401 | 303-275-3000 | www.nrel.gov

NREL is a national laboratory of the U.S. Department of Energy, Office of Energy Efficiency and Renewable Energy, operated by the Alliance for Sustainable Energy, LLC.

NREL/FS-5500-54219 • August 2012 